\title{
Automatic Vehicle Number Plate Recognition System using Matlab
}

\author{
Bhawna Tiwari ${ }^{1}$, Archana Sharma ${ }^{2}$, Malti Gautam Singh ${ }^{3}$, Bhawana Rathi ${ }^{4}$ \\ 1,2,3,4 (Assistant Professor, ECE Department, Krishna Engineering College, Ghaziabad, U.P., India)
}

\begin{abstract}
Automatic number plate recognition is a mass surveillance method that uses optical character recognition on images to read the number plates on vehicles. Existing closed-circuit television or road-rule enforcement cameras, or specifically designed systems can be used for the task. This system is very helpful for traffic police to find the details of a car violating the traffic rules. Its applications also includes Automatic toll collection system and car parking systems. [1] In high security areas where parking space is reserved for VIP vehicle owners only, the parking gate will be opened after number recognition. In areas where parking space is allotted to a particular vehicle, wrong vehicle parked can be recognized. ANPR can be used to store the images captured by the cameras and the text from the number plate. Systems use infrared lighting to allow the camera to take the picture at any time of day. A powerful flash can also included in cameras, to both illuminate the picture and make the offender aware of his mistake. Due to plate variation from place to place ANPR technology tends to be region specific.
\end{abstract}

Keywords: ANPR( Automatic Number Plate Recognition),CRS (Computer Recognition System),OCR (Optical Character Recognition), Vehicle Number Plate, Matlab

\section{Introduction}

Being able to identify vehicle number plates quickly and mechanically is beneficial for many businesses and organizations for a wide variety of applications including traffic management, automatic payment systems for car parking, security and crime detection. The ANPR was invented in 1976 at the Police Scientific Development Branch in the UK. Prototype systems were working by 1979 for industrial systems, first at Electronics then at Computer Recognition Systems (CRS) in Wokingham, UK. Various recognition techniques have been developed and number plate recognition systems are being used now a days in various traffic and security applications, such as parking, access and border control, or tracking of stolen cars. Automatic number plate recognition systems can be used in access control. For example, in many companies this technology is used to grant access only to vehicles of authorized personnel. In some countries, ANPR systems is used to automatically detect and monitor border crossings.

ANPR system is an image processing technology in which number plate of vehicle is used to recognize the vehicle. The objective is to design an efficient automatic vehicle recognition system by using the vehicle number plate, and to implement it for various applications such as automatic toll tax collection, parking system, Traffic control, etc. The system has four main steps to get the required information. These are image acquisition, Pre processing ( Number plate detection), character segmentation and character recognition. [2] This system is implemented and simulated in Matlab 2010a.

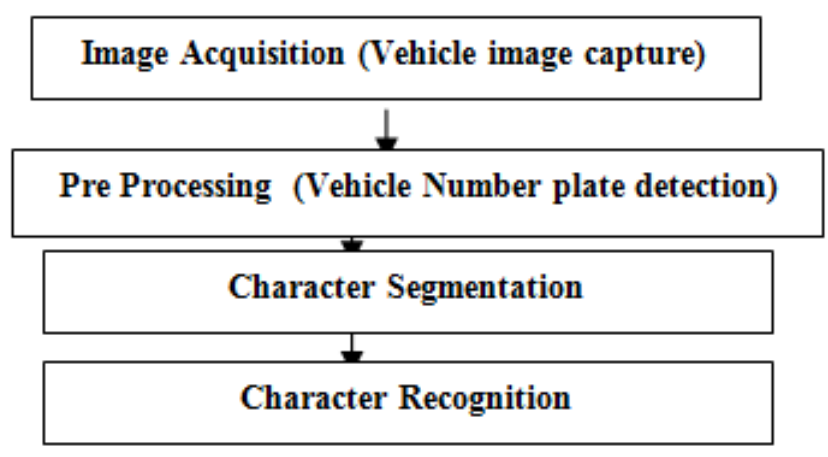

Fig 1 Flow chart illustrating stages of ANPR system

\subsection{Background}

Vehicle Number Plate Recognition is an image processing system which is used to recognize the vehicles by identifying the number plate. It is basically use for traffic control and security purposes. Firstly, the 
vehicle will stop at the car gate. The cycle will start when clicked on run button. It will activate the camera to take a snap shot.

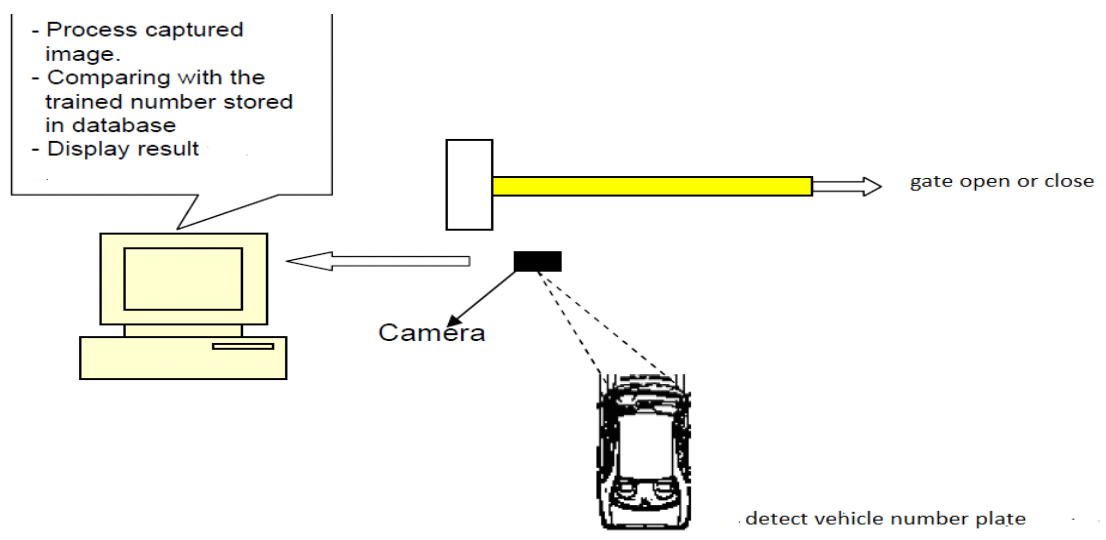

Fig. 2: Block Diagram of ANPR System

The system will apply certain algorithm to analyze the vehicle image. Then, the images will be enhance, locating the vehicle plate position and extract the characters from the vehicle plate. The system will try to match the recognized vehicle plate number with the vehicle number plate database. If "Access Granted", the gate will open and allowed the vehicle to pass through. The Vehicle Number Plate Recognition also provide an advantage by storing the image of the vehicle in which it will be useful for crime fighting. Speed of the vehicle, weather condition, type of Vehicle, distance between vehicle number plate and the camera, type of plate (Rectangular, Bent type), Vehicle number plate orientation and type of vehicle font character are the variables that affect efficiency and accuracy accuracy of recognition system.

Since the license plate normally has a rectangular shape with a known aspect ratio, it can be extracted by finding all possible rectangles in the image. Edge detection methods are commonly used to find these rectangles [3] [4]. In [4], Sobel filter is used to detect edges. Candidate regions are generated in [2], by matching between vertical edges only. In [4], the vertical edges are matched to obtain some candidate rectangles.

\subsection{Objectives}

The overall objective of the paper is to develop a system that recognize vehicle number plate from a car at a gate entrance of a parking area. The software could lead to a less expensive and faster way of enhancing and determining the performance of the recognition system. The system will generate report on the vehicle number plate it has captured. Once the vehicle number plate is captured, the characters will be recognized and displayed on the screen.[5]. Apart from this, the system can also serve as a security purpose whereby it can spot on any wanted or stolen vehicles. Earlier it was implemented but had less accuracy. Thus, there is need to address a set of constraints and focus on the design of the algorithm to extract the vehicle number plate in order to improve the accuracy and efficiency.

\section{Number Plate Recognition Using MATLAB}

Proposed Automatic Car Number Plate Recognition System mainly focusing on red light jump. If a vehicle jumps a red light the sensors are placed on which detect the presence of vehicle. As the vehicle passes the sensor, the camera will automatically capture the image using MATLAB image processing. Then the image is processed and the number from it will be extracted. Using the information, registered vehicle number will matched with the numbers of predefined record we will be able to find the details of the owner who broke the red light. Then record of rule brokers will be maintained and challan will be entered in those record and can be punished. This system can also be implemented on all rules and types of vehicle can also be detected. MATLAB is chosen for the number plate recognition application. The task is to construct the algorithm and recognizes can be done using MATLAB. MATLAB is very efficient as it have built-in-function tools for neural network and image processing. The advantages of MATLAB can be classified as follows: Platform independence, predefined function and device-independent plotting, Graphical User interface and MATLAB compiler.

It integrates computation, Algorithm development, Modeling simulation, and prototyping ,Data acquisition, Data analysis, exploration, and visualization and Application development, including graphical user interface building. MATLAB is high performance programming language which has an easy-to-use environment where problems and solutions are expressed in familiar mathematical notation. 


\subsection{Image cropping}

\section{Software Implementation}

Image cropping is a recognition process whereby it will extract the smallest rectangle which will contains the edge of the number plate and number plate itself. As the number plate surrounding is not so important, this cropping process will highly increase the speed of image processing. In order facilitate the next process smoothly and reducing the processing time, the image obtain is being converted to Binary Image by using the toolbox in MATLAB .[6][7]

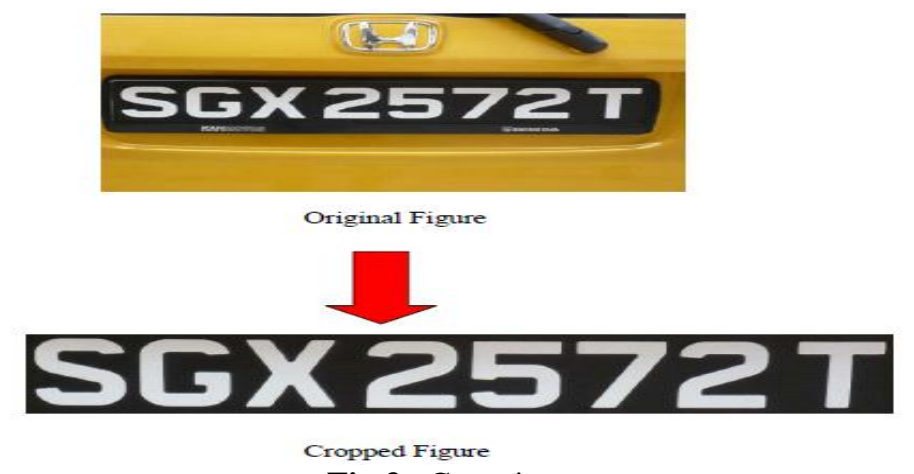

Fig 3 Cropping

\subsection{Binary Images}

It is an image which quantised into two values representing 0 and 1 or in pixel values of 0 and 255 representing the color black and white. Binary Images are obtained by converting the input image into grayscale format, then by converting the grayscale image to binary image by thresholding. The pixel of grey level above the threshold will be set to 1 (equal to 255; white) and the rest will be set to 0 (black). We will obtain white object with black background or vice versa. [6]

\subsection{Image enhancement}

Image enhancement is used to increase and improve the visibility of the image. Image Enhancement techniques consists process of sharpening the edges image, contrast manipulation, reducing noise, color image processing and image segmentation as well. [7]

\subsubsection{Histogram processing}

The Histogram image equalization is the process whereby the preceding transformation will generate an image that will show a histogram at each intensity level. The net result of the process will yield an image with an increased sparse range of intensity and higher contrast compare to the original image. The processed image from the histogram is not uniform due to the discrete nature. Histogram equalization produced an adaptive transformation function and the histogram will not change unless there is any change in the image.

\subsubsection{IM complement}

IM complement will compute the complement image (IM).IM can intensity, binary or true color image. For the complement binary image, black will becomes white and white will become black; zeros will become ones or ones will become zeros.

\subsection{Thresholding}

Thresholding is one of the oldest segmentation method. One of the simplest process will be the Graylevel thresholding. The value of threshold (T) is being selected and compare with the pixel of the image. It also transform the input image $(\mathrm{K})$ into an output binary image $(\mathrm{F})$ which is being segmented.

$\mathrm{F}(\mathrm{x}, \mathrm{y})=1$ if $\mathrm{K}(\mathrm{x}, \mathrm{y})>=\mathrm{T}$;

$=0$ if $\mathrm{K}(\mathrm{x}, \mathrm{y})<\mathrm{T}$

Representing F $(\mathrm{x}, \mathrm{y})=1$ for image object; $\mathrm{F}(\mathrm{x}, \mathrm{y})=0$ for background of the object and $\mathrm{T}=$ threshold.

\subsubsection{Global thresholding}

In global thresholding, the histogram of the image is being partitioned using a single threshold value. The threshold define the value of the gray level in which the baseline boundary is in between the foreground pixels and the background. . 


\subsubsection{Local thresholding}

In local thresholding ,partitioning the image into sub-images and the value of threshold vale is determined from this sub-images.

\subsubsection{Optimal global thresholding}

It approximate the threshold and produce in minimum error segmentation. It depends on the approximation of the image histogram in which it used the function of the overall densities.

\subsubsection{Adaptive thresholding}

By using the adaptive thresholding, the value of the threshold is computed for every pixel separately using the neighborhood. If the histogram is bimodal, the value of the threshold will be taken in the middle of the bimodal. For other cases, in which the modes are overlapping,, the threshold will try to reduce the error of taking the background pixels as object pixel and vice versa.

\subsection{Edge detection}

The initial step in recognition of vehicle number plate is by detection of the number plate size. The challenge is to include an algorithm that are able to detect the rectangle plate in the image. Let's define the rectangular plate as the horizontal and vertical edges. The horizontal and vertical edges of high density is normally caused by the contrast character from the number plate and this will eventually lead to detecting the wrong area.[6][7]

Edge detection is used to find the transition between the two area based on the discontinuities in the intensity values.. Edge detection also base on the image edge information which is found by the relation of the pixel and its neighbour. The magnitude of the vector representing $\mathrm{f}$ is an important quantity to the edge detection where $\mathrm{f}$ is given by:

$$
\nabla f=\operatorname{mag}(\nabla f)=\left[G_{x}^{2}+G_{y}^{2}\right]^{0.5}
$$

The vector for the gradient of the image $f(x, y)$ at location $(x, y)$ is given by:

$$
\nabla f=\left[\begin{array}{c}
G x \\
G y
\end{array}\right]=\left[\begin{array}{c}
\frac{\delta f}{\delta x} \\
\frac{\delta f}{\delta y}
\end{array}\right]
$$

\subsubsection{Image noise}

Digital camera with long exposure time setting and high ISOs will produced images with lots of noise. Noise in the images is appears as the colour speckles. There will be not enough light source for proper exposure to activate in a low light area. Therefore, longer time is needed for the image sensor for collection of weak signal. In this circumstance, the electrical noise from the background will be higher than the signal.From the amplification of the signal, the electrical noise in the background is also being amplified

\subsubsection{Filtering}

In this pre-processing stage, filtering is used for blurring and for noise reduction and thus resulting a more sharpen image. Blurring involved in removing and cleaning up of small patches and details from the image prior to object extraction, bridging of gaps in curves and lines. Blurring with liner/non liner filter is able to achieve noise reduction. In order to reduce the background noise, softening is often applied to the low pass filter and it will affect the image to become blurring. High pass filter is used to determine and intensify the details in the image, but noise will be added during the process. High pass filter also used the convolution during the process.

\subsubsection{Erosion}

Erosion is one of the simplest method used for removing unwanted details from a binary image. There are also new method versions of erosion that work on grayscale images as well. The process of erosion basically reduces the objects size and getting rid of unwanted details by encoding the image with a structuring element of radius that is smaller than the object. 


\subsubsection{Filtering of digits}

Now the black and white image of the Vehicle number Plate can be obtained.. It has been cropped to a rectangular shape in which it enhances the algorithm to be more uniformed in the further stages. The non-digits substance which appears in the image is caused by the noise (quantization that will transform to 'Black'), object or substance which is not digits/characters such as screws, sand, water droplet, frame lines, effects on JPEG compression.

By Filtering, the unwanted substances or noise can be removed or filtered out that is not a character or digits. Lastly, the image is only left with characters and digits in which we are interested. The 2 stages for the algorithm is as follows:

1) Remove out the small objects or connected components.

2) Identify the frame line that is connected to the digits and separate it.

Identify and remove away the small connected objects

For this stage, we need to identify the connected components. The component can have a value of either 4-connected or 8-connected. In this algorithm, we use 8- connected (8 specify 8-connected objects). After the component has been labelled, they will obtain a unique number; the elements of labels1 (stated in the algorithm) contains integer values that will be greater than or equal to 0 . The pixel of 0 represents the background and pixel of 1 will make up the first object, the pixel of 2 will label the second object, so on and so for.

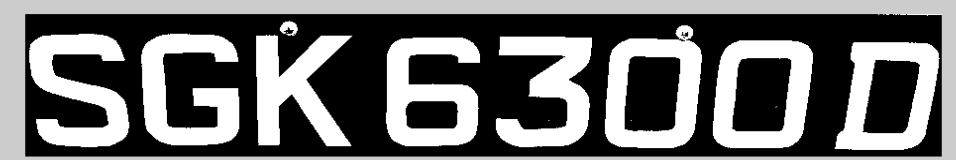

Fig 4 Illustrate an example of filtering process.

For the above image, 29 connected components are found in the BW image. An algorithm is created whereby the threshold is determined by doing a statistic check. Results obtain before filtering numOfDigits $1=29$

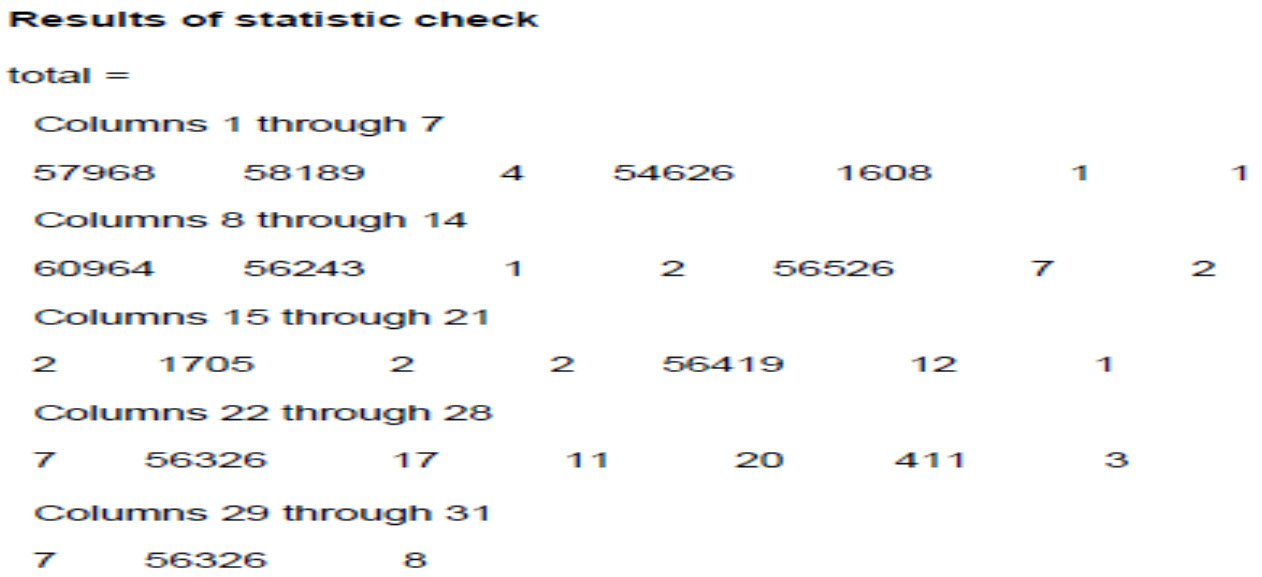

Next, bwareaopen (Image Processing Toolbox) is applied which will remove all the connected components from the binary image that have value less than $\mathrm{P}$ pixels, in which it will produce another binary image. Therefore, components with less than 54626 is classify as non-digits components and need to be taken away.

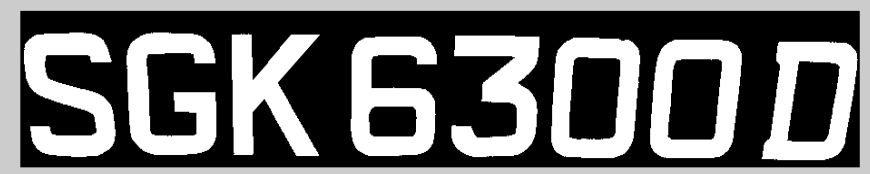

Fig 5. Results after filtering 


\subsection{Image segmentation}

In order to extract and analyzed the object characteristic, image segmentation is used , the process which need to partition the image into different parts that will have a strong correlation with the objects.

Segmentation process can be categorized into several parts. Firstly is the global knowledge of an image. The feature of the image is represented by a histogram. Secondly, in the boundary-based segmentation.,edge detection is used to obtain the region contours and the objects will construct from the obtain contours. Another one is edge-based segmentation. For this paper, first category segmentation method is being used which is by using threshold.

\subsubsection{Feature extraction from digital image}

The digital image description is based on the external and internal representation. The color or texture of the image is the internal representation whereas the external representation is based on the characteristic of the shapes of characters. The descriptor vector includes the characteristics as the number of lines, vertical or diagonal edges etc. The process of the feature extraction is to transform the bitmap data into a form of descriptor in which more suitable for computer. If we classify the similar character into classes, the descriptor of the character from the same class is close to each other in the vector space and this will lead to a success in pattern recognition process.

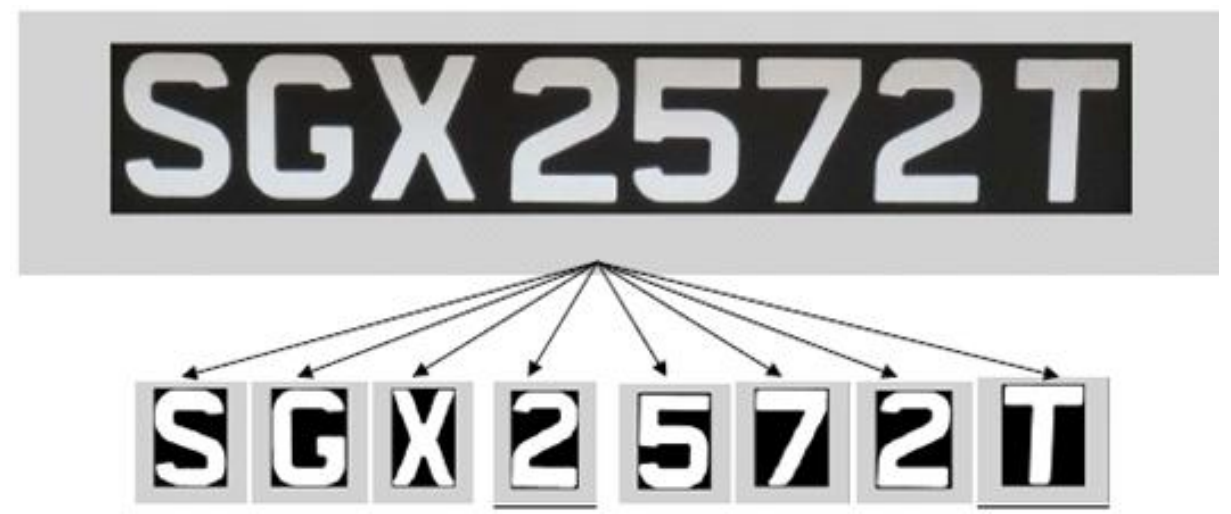

Fig6. Feature Extraction

\subsubsection{Database}

It is a collection of information or data which it is being orderly organize, thus it can be accessed easily and updated. Database can be in the form of text, contents and images and is needed to make sure that the image space can contained enough characters which have been extracted and the vehicle number plate number stored in the excel sheet for the purpose of comparison. The database would be large enough in order to improve the accuracy and better chances of obtaining the correct result. The class recognition will check if the computed ratio is correct. It will ignore the class if the image thresholding is too low. If the class has match correctly, it will be compare with data set in the predefined database and provide an output if the image is recognized correctly.

\subsection{Character recognition}

In this step, the extracted characters are resized to fit the characters into a window. For the paper, each character is normalized to the size of $(50 \times 30)$ binary image and then follow by reshape to standard dimension before sending the data set to neural network for training.

\subsection{Development of the proposed recognition sytem simulation}

The paper will begin by reading the objectives set at the initial stage of the report. Subsequently, the simulation of the specification will be studied. Software for simulation will be developed in the modular form for testing and to troubleshoot more easily. The modules of development is listed in the three main parts i.e. Extract individual digit from the vehicle plate, Recognize individual digit and Check with database.

\section{Conclusion}

The development of the Automatic Number Plate Recognition System is being implemented using MATLAB by using following listed out process.

i. Read the color image into MATLAB

ii. Manually crop the license car plate region from the color image

iii. Analyze the cropped image in its red, green blue frames 
iv. Convert the image from RGB to HSV and analyze its Hue, Saturation and Value

v. Image enhancement by using Histogram Equalization

vi. Image quantization by using thresholding

vii. Noise Reduction

viii. Extract each character and number from the image using segmentation techniques

ix. Results is to be displayed in Matlab

Details of vehicle's owner displayed on screen shown below:

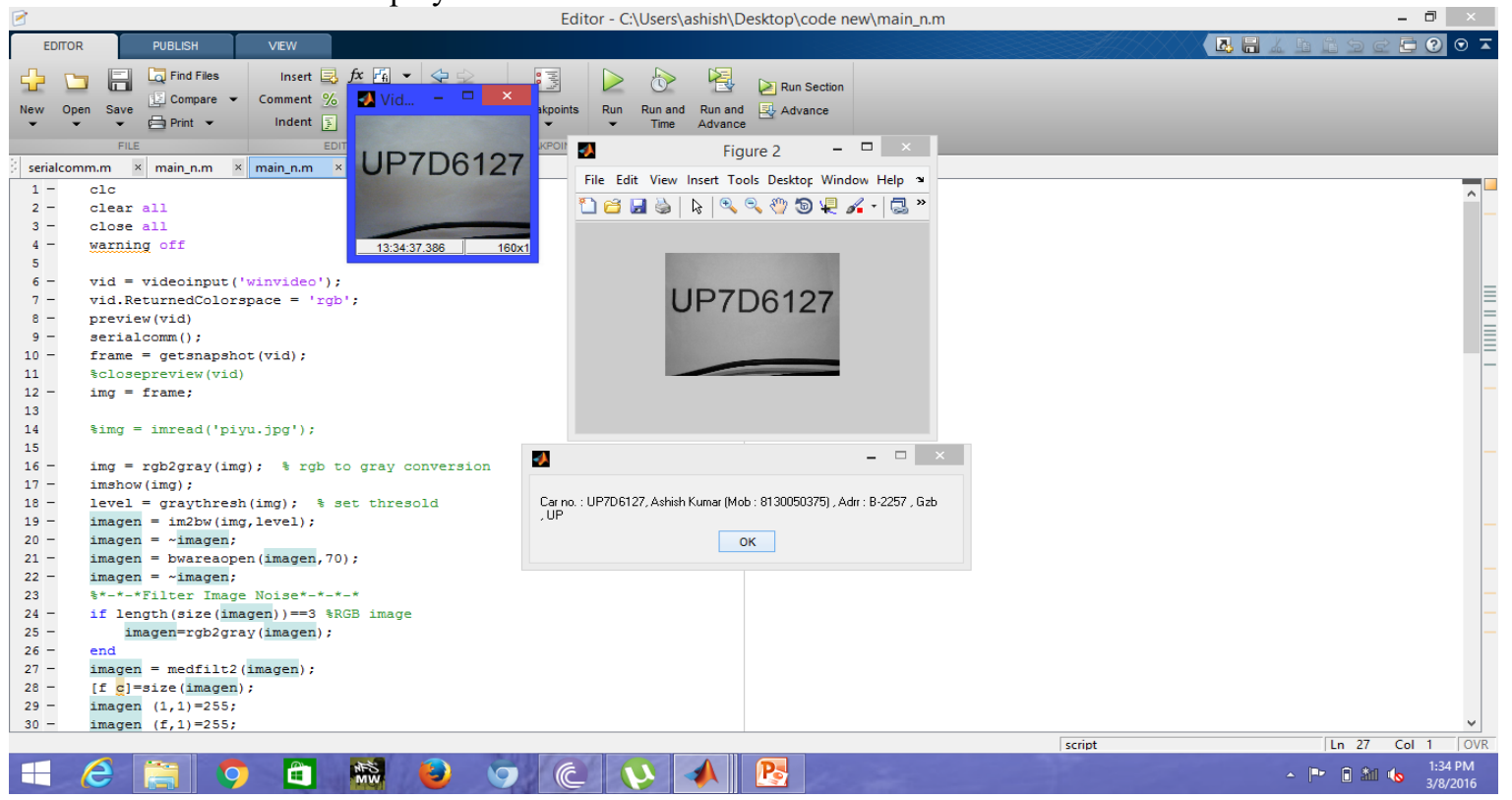

Fig 7 Vehicle Owner details result

\section{References}

[1]. Amr Badr, Mohamed M. Abdelwahab, Ahmed M. Thabet, Automatic Number Plate Recognition, Mathematics and Computer Science Series, Vol 38(I),2011, 62-71

[2]. DhirajY. Gaikwad, Promod B. Borole, A Review Paper on Automatic Number Plate recognition ( ANPR) Sytem, International journal of Innovative Research in Advanced Engineering, Vol I Issue,2014

[3]. H. Bai and C. Liu, A hybrid license plate extraction method based on edge statistics and morphology, Proc. Int. Conf. Pattern Recognit., vol. 2. 2004, pp. 831-834.

[4]. F. Faradji, A. H. Rezaie, and M. Ziaratban, A morphological-based license plate location,IEEE International Conference of Image Processing, vol.1. Sep.-Oct. 2007, pp. 57-61

[5]. Mritunjay Singh, A New and Efficient Method for Vehicle License Plate Detection, International Journal of Advanced Research in, Computer Science and Software Engineering 3(12), December - 2013, pp. 1002-1006

[6]. Rafael C. Gonzalez, Richard E. Woods and Steven L. Eddins, Digital Image Processing using MATLAB

[7]. Rafael C. Gonzalez and Richard E. Woods, Digital Image Processing Second Edition.

[8]. C. N. E. Anagnostopoulos and I. E. Anagnostopoulos, A License Plate Recognition Algorithm for Intelligent Transportation System, Applications, IEEE Transaction on Intelligent Transportation System, Vol. 7, No. 3, 2006, pp. 377-392. 